\title{
Vehicle Lane Detection and Tracking System for Car Safety
}

\author{
Prof. A. S. Jahagirdar \\ MIT College of Engineering, \\ Pune,India \\ (aditi.jahagirdar@mitcoe.edu.in)
}

\author{
Prajakta Yelwande \\ Dr. Vishwanath Karad MIT World Peace \\ University, \\ Pune, India \\ (praju.21995@gmail.com)
}

\begin{abstract}
Advanced Driving Assistance Systems(ADASs) is important technology for safety of car as well as car driver and lane detection is a main purpose of current Advanced Driving Assistance Systems (ADASs). Most of the previous results focuses on the study of vision-based methods. In this paper, previous lane detection methods and algorithms are reviewed. Further, the limitations are studied and analyzed to evaluate the more robust detection systems and to improve the accuracy of detection system.
\end{abstract}

Index Terms-Advanced Driving Assistance Systems(ADASs), lane detection, computer vision, accuracy, complexity, data mining, Hough transform, edge detection, Region of Interest(ROI), vehicle detection, canny algorithm, sobel operator.

\section{INTRODUCTION}

According to survey of World Health Organization(WHO), in china more than 200,000 people expire each year because of road accidents and car crashes. Advanced Driver Assistance Systems(ADASs) is useful for lane detection. Visionbased ADASs are used for lane detection as well as lane departure system. Now a days, detecting road lanes accurately is an important issue. Lane boundaries enables vehicles to stay in the proper lane and avoid car crashes.

The typical method for lane detection consists of four main steps: Pre-processing, feature detection, fitting and tracking. In pre-processing step, a system removes noise and prepares images for the further steps. Feature detection consists of two methods: lane-feature based method and geometric information based method. To detect straight lines, Hough transform and related algorithms are used. To detect the lane mark, traffic signs, traffic light, obstacles, camera sensor is used on an autonomous vehicle. Lane detection method often follows a process with the following steps:

- Capture a image from the camera sensor

- Convert the original image into grayscale image

- Threshold the image

- Find the lane mark by lane line

- Use filter
Using above steps, most of the researchers usually focuses on improving the accuracy of the detected lane mark, increase detection speed and reduce complexity.

\section{BACKGROUND STUDY AND LITERATURE OVERVIEW}

\section{A. Background}

Most of the time accidents are caused by lack of concentration and not maintaining a safe car distance to the car in front, or changing lanes without paying attention for vehicles which is next to the car. This project is about detecting the boundaries of the lane and to inform the driver if he/she is going to change the lane without indicating for his/her intention. The system should also try to measure the distance to the vehicle in front of that vehicle and signalize if the distance in not safe enough.

\section{B. Literature Overview}

Safety of vehicle as well as driver's safety are important issues are considered in addressing national as well as international road issues to achieve a safer and efficient road traffic system. Vehicle safety plays an important role for safety of all road users and also useful to measure the crash avoidance or reduction of injury. The purposes of Advanced driver assistance systems are to reduce the risk and assist post impact care are also investigated for future application.

New technologies have the capability to increase risk and decrease crash and injury risks simultaneously. The services provided in this project are given below:

- To detect the lanes on road and to inform the driver if he/she is going to change the lane without indicating for his/her intention.

- To indicate driver while changing the lane

- To measure the distance between two cars to avoid car crashes. 


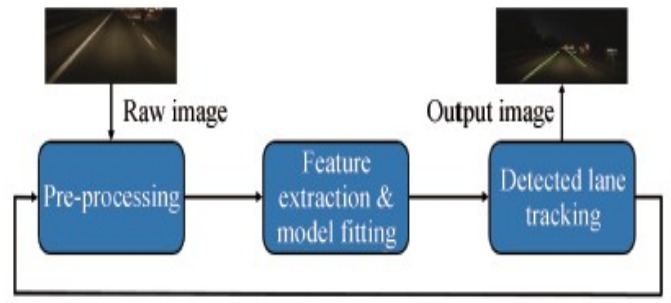

Fig. 1. General architecture of lane detection system. The loop shows that the tracked position of the lane markings after detecting the lanes will be used for further processing.

[2] In above figure, once the input image is pre-processed, lane features such as colours and edge features will be extracted. To detect the straight lines, hough-transform method is used. After detection of lanes successfully, kalman filter is used to find the position of lane markings.

\section{RELATED WORK}

[1]states that vision based ADASs are mainly useful for lane marking detection, lane departure warning, identification of traffic signs, traffic barrier detection, pedestrian detection and intelligent cruise control. Lane detection method consists of four steps:

- Pre-processing

- Feature detection

- Fitting

- $\quad$ Tracking

In pre-processing step, a system removes noise and generates images for development of subsequent steps. In feature detection step, system can be divided into two classes i.e feature based and geometric information based method. In this system, kalman filter is used in tracking phase to track the two sides of the lane markings. CNN classifier removes the non-lane regions. To facilitate the $\mathrm{CNN}$ classifier, local waveforms are generated to extract regions of interest (ROI).

Lane structures are analyzed using three stages:

- Waveform Generation: Waveform depicts the voices of moving objects.

- Convolutional NNs: In this, CNN classifier is trained to eliminate non-lane regions.

- Geometric Analysis: Geometric analysis of lane markings are judged in the vertical and horizontal directions.

[2] tells about previous vision-based lane detection methods in three parts:

- Lane detection algorithms

- Integration

- Evaluation methods

The utilization of vision-based techniques detects lanes from the camera devices and prevents the driver from making unintended lane changes. ACP is short for Artificial Society, Computational experiments, Parallel execution. Vision-based lane detection systems consists of three procedures: image processing, lane detection, lane marking. In this, pre processing plays an important role in region of interest(ROI) selection, vanishing point detection, transferring color image into grayscale image or a different color format, noise removal, inverse perspective mapping (IPM) etc.

[3] proposed a system in which a single RGB$\mathrm{D}$ camera is attached to the vehicle which is used to collect data of the environment. Further recorded images are converted into binary images. In this, principal of component analysis(PCA) technique is used to refine the templates. This paper is classified into four parts:

- Image pre-processing

- Respond map computation

- Template enhancement

- Lane model fitting

- In image pre-processing, data from RGB channels are combined and converted to a eight-bit grayscale image. Further this image is converted to a half binary format by calculating a threshold value.

- In respond map computation, using colour and depth of images, next step is to compute for each pixel a probability that it belongs to the left or right marker. A combination of all probabilities is called respond map.

- In template enhancement, principal component analysis (PCA) technique is used to refine the templates with highest probability.

- In lane model fitting, RGB-D matching method is useful to remove the outliers.

[4] proposed an algorithm which is based on two approaches: Median strip detection and lane change detection.

In median strip detection approach, static objects like a barrier, small wall, green grass etc are identified at an known maximal longitudinal distance. In lane change detection approach, knowledge of the vehicle distance from the left and right lane boundaries is useful to determine the instants at which vehicle moves to a different lane.

[5]focuses on lane detection(LD), and lane tracking (LT) problems arising in lane departure warning systems(LDWS). LDWS consists of two functions and two devices ie. cameras, longitudinal and angular rear wheel speed sensors, LD and LT software modules, lane departure warning module. Basically LDWS algorithm has three steps:

- Lane detection

- Lane tracking

- Lane departure

In lane detection, image pre-processing technique is used to improve the image contrast, reduce the image noise etc. Image smoothing filters are used to reduce the noise and to blur the image. Laplacian is also used for 


\section{Asian Journal of Convergence in Technology ISSN NO: 2350-1146 I.F-5.11}

edge detection. Canny's algorithm plays an important role for edge detection. Canny set has three criteria:

- Ability to detect the edge

- Ability to locate the edge

- Uniqueness of the solution

Hough transform is also used for straight line detection. It has five steps:

- Capture RGB image

- Grayscale conversion

- Hough's transform

- Accumulation matrix calculation

- Local maxima search

[6] proposes the Reduced Resolution Lane Detection algorithm. This algorithm consists of two modes i.e. the iteration mode and the color threshold mode. Iteration mode is used to find the lane marks by iterating the image processing threshold values and then detects the lane marks. Color threshold mode is used to filter out noise by color threshold. After reducing the resolution of threshold images, to detect the lane pixels, lane pixel detection and filtering method is used.

[7] proposes a real time lane detection and tracking algorithm based on Gabor filter and edge extraction. Gabor filter is used to enhance the characteristics of the lane. Initially Gabor filter can capture the local structure information corresponding to the spatial position, spatial frequency. It can extract the edge information in multi-direction and multi-mode. To find the more accurate edge direction information, sobel operator is used to smooth the noise.

[8] proposes a novel spatial-temporal incremental clustering(STIC) algorithm with curve fitting to detect and track lanes in real-time. The purpose of a lane detection system is to detect the lane boundaries and warn the driver if the vehicle is going too close to the boundary. This system works well for various types of lane markings such as continuous or non-continuous as well as straight or curved roads.

This paper is divided into five parts:

- $\quad$ Region of Interest(ROI)

- Grayscaling and Noise Reduction

- Segmentation of foreground regions

- Connected component detection

- Spatio-Temporal Incremental Clustering (STIC) for lane detection

By selecting a region of interest, system should work fast and efficiently so it is necessary to reduce the computational time. The ROI can be converted from RGB to grayscale for improving the segmentation of the lane markings. The main purpose of segmentation is to segment the ROI into relevant and non-relevant areas. Adaptive thresholding is used to convert the ROI into a binary image. To find the orientation of connected components, principal component analysis technique is used on each of connected components. Using STIC, connected components are classified into small and big connected components after detecting.

\section{Volume V Issue I}

In [9] Road lane detection technique is developed based on an on-board PC-based vision system. PC-based vision system and laser range scanner are installed on a four wheels drive electric vehicle to construct a lane detection and following control system. The FPGA control structure is used to monitor the vehicle motion, It also avoids the obstacles.

[10] proposes a lane detection method which consists of following steps:

- Pre-processing: In this stage, RGB image is transformed into gray image and region of interest is selected.

- De-noising: The noise is removed and the frame is processed using filtering operation.

- Edge detection: Each pixel in the ROI is selected for strong intensity gradients as that the edges of the image elements.

- Removal of outliers: This operation helps in removing small outliers and fills the gap between the lane lines.

- Lane detection: In this stage, Hough transform is used as it performs the segmentation and fitting line process.

The main objective of this paper is to use image processing techniques to identify the lane lines on the hilly road based on Hough transform. This paper also helps to detect the lane lines on straight and curved roads.

[11] proposed an approach to detect information about lanes and vehicles for driver assistance or warning system. In this study, camera is installed on the test vehicle. Images from the camera can detects three lanes (i.e. centre, left and right boundaries) and multi-vehicles.

In lane detection, line detection and inverse perspective mapping are used. For the vehicle detection, horizontal and vertical edge detection are combined. The horizontal edge is used to detect the vehicle candidates and then the vertical edge detection is used to verify the vehicle candidates. In this paper the key idea is to integrate the relationship between lanes and vehicles to support the warning system or a driver assistance system. This paper is divided into two parts:

- Lane detection

- Vehicle detection

[12] proposed a approach which is developed in order to define a safe and optimal trajectory to be tracked by a road vehicle. The lane boundaries are extracted using a vision-based lane detection algorithm. This paper addressed the problem of the generation of an optimal constrained lane reference to be tracked by the automated guided vehicle. A vision-based lane detection algorithm is therefore employed to detect lane boundaries that present the constraints for the optimal path. Lane boundaries detection uses homography transformation because the captured images includes unnecessary data which may cause faulty lane detection. homography transformation is useful to reduce the computational time by eliminating the 


\section{Asian Journal of Convergence in Technology ISSN NO: 2350-1146 I.F-5.11}

undesirable road parts. After the homography transformation of the captured road image, a filtered image segmentation approach is used to detect the lane boundaries.

[13] presents an efficient, robust Lane Departure Warning System(LDWS) and a frontal collision warning system. This system uses a monoscopic dashboard camera fitted in the wildshield of the car. This system has three components:

- Lane detection

- Vehicle detection

- Distance Estimation

In lane detection, Inverse Perspective Mapping(IPM) of the ROI is taken using homogeneous transform. Canny edge detection mechanism is used to find the vertical edges. Then Hough-transform is applied on the top of these points to find the long parallel lines that are nearly vertical. In vehicle detection, the ROI is smoothened with a Gaussian filter. It is then thresholded and canny edge detector is applied. In distance estimation, after detecting the vehicle for consecutive frames, the presence of vehicle is confirmed.

[14] proposed a new detection method to predict a vehicle's trajectory and used it for detecting lane changes of surrounding vehicles. The proposed method defines three types of features: the distance from the centerline, the lateral velocity and the potential feature. The sensor system is installed in the primary vehicle. It consists of a position sensor and six laser scanners. For driving-intention estimation, feature vector as consisting of the distance from the centerline, the lateral velocity and the potential feature are defined.

In [15] a method of lane edge detection based on Canny algorithm is proposed to get an ideal edge of the lane markings in road image. Characteristics of road imagr are analyzed and road image is divided into subregion 1, subregion 2, subregion 3. Canny edge detector is known as the three performance criteria for edge detection:

- Good detection

- Good localization of edge points

- Only one response to a single edge

In [16] True flow as an addition to conventional optical flow is introduced. Translational and rotational influence of ego motion is eliminated, allowing to estimate the observed object behaviour.

The presented approach consists of three steps:

- An object reference point is determined within the image to derive its position relatively to the visible lane.

- Camera motion between consecutive frames is determined and the resulting motion field at the reference point is removed from optical flow.

- Utilization of examined flow to extract invariant features.

In [17] robust lane estimator is proposed for lane based target selection. The lane estimator is designed with clothoid road model and an assumption based on lane www.asianssr.org

\section{Volume V Issue I}

width. To verify the performance of proposed lane estimator, realcar test in the public road is carried out. Kalman filter is useful for detecting the edges of the lanes.

[18] gives the review of lane detection methods and the lane departure system methods. Lane detection is based on two approach such as feature based and model approach. The feature based approach mainly focuses on the quality of the images and the model based approach requires the inverse perspective mapping.

[19] analyzes the edge position detection method for segmental wireless power supply for electrical vehicles without position sensors. A higher phase angle varying range (PAVR) is preferred to achieve a better position judging. This paper also introduces a novel method to decide whether the vehicle is approaching a segmental track coil without position sensors.

[20] proposed a real-time and robust lane and leading vehicle detection and tracking system. The whole system is combined with lane detection and tracking, sky and road region recognition, vehicle detection based on convolutional neural networks and vehicle tracking. Hough-transform is used to extract straight lines on the road. Finally the inter frame information is used for lane tracking to achieve realtime and stable lane detection.

[21] proposed a simple method to anticipate the relative position of the vehicle in the following frame from the current frame image. The expected ratio between a vehicle and left-right lanes can be obtained by using of the speed of a vehicle and the frame speed of a camera. SAP based algorithm can predict the speed-adaptive lateral ratio between left and right lanes.

In [22] an anomaly detection technique is used to compare snapshots of normal and dangerous lane change maneuvers, to identify the abnormal instances. A one-class support vector machine is used for novelty identification of naturalistic driving study data. The goal of this paper is to distinguish between normal and abnormal lane change instances.

In [23] A new positioning system based on RFID and vision is proposed. This system aims at lanelevel positioning even in dense urban areas. In this system, vehicle positioning is divided into two processes:

- Judging the specific lane the vehicle is occupying

- Obtaining the absolute position or relative position to other vehicles

In [24] system is based on single camera sensor. For lane detection, a modified inverse perspective mapping using only a few extrinsic camera parameters. In lane tracking kalman filter and particle filter based approach is used. For localising vehicle on the road estimation of some related parameters like its current lane, shape of the road and its position from centreline. To compute this parameters the road environment is scanned using wide angle camera sensor and extract lane markers. 


\section{Asian Journal of Convergence in Technology ISSN NO: 2350-1146 I.F-5.11}

[25] proposed a recognition algorithm using HSV color space and lane shape feature to detect the lanes. This algorithm is useful for autonomous driving and accident prevention. Progressive Probabilistic Hough $(\mathrm{PPH})$ is used to determine final lane.

\section{CONCLUSION}

In this study, Vision based lane detection systems are reviewed. We have studied the different approach of lane detection and lane departure system. Reduced Resolution algorithm is also studied for improving the lane detection processing speed. In this paper, we have discussed about a method of lane edge detection based on canny algorithm.

\section{REFERENCES}

[1] Yang Ye, Xiao Li Hao, Hou Jin Chen, "Lane Detection method based on lane structural analysis and CNNs", IET Transp. Syst., vol. 12, Iss. 6, pp. 513-520, 2018.

[2] Yang Xing, Chen Lv, Long Chen, Huaji Wang,Hong Wang, Dongpu Cao, Efstathios Velenis, Fei-Yue Wang "Advances in visionbased Lane Detection: Algorithms, Integration, Assessment, and Perspectives on ACP-Based Parallel Vision", IEEE/CAA JOURNAL OF AUTOMATICA SINICA, VOL. 5, NO. 3, MAY 2018.

[3] Cong Hoang Quach, Van Lien Tran, Duy Hung Nguyen, Viet Thang Nguyen, Minh Trien Pham, Manh Duong Phung "Real-time Lane Marker Detection Using Template Matching with RGB-D Camera", 2018 2nd International Conference on Recent Advances in Signal Processing, Telecommunications Computing (SigTelCom).

[4] Lioris,Annie Bracquemond, Gildas Thiolon, Laurent Bonic, "Lane Change detection algorithm on real world driving for arbitrary road infrasructures", 2nd IEEE International Conference on Computer Software Applications,2018.

[5] Gianni Cario, Alessandro Casavola, Marco Lupia, "Lane Detection and Tracking Problems in Lane Departure Warning System", 2017

[6] Li Dang, Girma Tewolde, Xiaoyuan Zhang, Jaerock Kwon, "Reduced Resolution Lane Detection", IEEE Africon Proceedings, 2017.

[7] Qi Gao, Yan Feng, Li Wang, "A Real time Lane Detection and Tracking Algorithm", IEEE, 2017.

[8] Any Gupta, Ayesha Choudhary, "A Real-time Lane Detection Using Spatio-Temporal Incremental Clustering", IEEE 20th International Conference on Intelligent Transportation Systems (ITSC),2017.

[9] Shiuh-Jer Huang, Chien-Chang Tsai, "Vehicle Lane Detection and Following Based on vision system and Laser Scanner", Proceedings of the IEEE International Conference on Applied System Innovation, IEEEICASI 2017 - Meen, Prior Lam (Eds), 2017.

[10] Kodeeswari M,Philemon Daniel, "Lane Line Detection in Real Time Based on Morphological Operations for Driver Assistance System", 4th IEEE International Conference on Signal Processing, Computing and Control (ISPCC 2k17), Sep21-23,2017,solan,India 2018.

\section{Volume V Issue I}

[11] Van Quang Nguyen, Changjun Seo, Heungseob Kim, Kwangsuck Boo, "A study on Detection method of vehicle based on lane detection for a Driver Assistance System using a Camera on Highway", 2017,11th Asian Control Conference (ASCC) Gold Coast Convention Centre, Australia,December 17-20, 2017.

[12] Kawther Osman, Jawhar Ghommam, Maarouf Saad, "Vision Based Lane Reference Detection and Tracking Control of an Automated Guided Vehicle", 2017, 25th Mediterranean Conference on Control and Automation (MED) Valletta, Malta,July 3-6, 2017.

[13] Vinuchandran A V, Shanmughasundaram R., "A Real-Time Lane Departure Warning and Vehicle Detection System using Monoscopic Camera", 2017 International Conference on Intelligent Computing, Instrumentation and Control Technologies (ICICICT).

[14] Hanwool Woo, Yonghoon Ji, Hitoshi Kono, Yusuke Tamura, Yasuhide Kuroda, Takashi Sugano, Yasunori Yamamoto, Atsushi Yamashita, Hajime Asama, "Lane Detection Based on VehicleTrajectory Prediction", IEEE ROBOTICS AND AUTOMATION LETTERS. PREPRINT VERSION, JANUARY, 2017

[15] Xuqin Yan, Yanqiang Li, "A Method of Lane Edge Detection based on Canny Algorithm", IEEE,2017.

[16] Joran Zeisler, Fabian Schonert, Marcel Johne, Vladimir Haltakov, "Vision Based Lane Change Detection using True Flow Features", 2017 IEEE 20th International Conference on Intelligent Transportation Systems (ITSC): Workshop.

[17] Moon-Hyung Song, Chang-il Kim, Jong-Min Kim, Kwang-Soo Lee, Hyun-Bae Park, Jae-Seok Jeon, Su-Jin Kwag, Moon-Sik Kim, "A Novel Approach to the Enhancement of Lane Estimator by using Kalman Filter", 2017 17th International Conference on Control, Automation and Systems (ICCAS 2017), Ramada Plaza, Jeju, Korea,Oct. 18-21, 2017.

[18] Suvarna Shirke, Dr. C. Rajabhushanam, "A Study on Lane Detection Techniques and Lane Departure System".

[19] Qijun Deng, Jiangtao Liu, Dariusz Czarkowski, Mariusz Bojarski, Jing Chen, Wenshan Hu, Hong Zhou, "Edge Position Detection of on-line charged vehicles with Segmental Wireless Power Supply", IEEE 2016.

[20] Huang Guan, Wang Xingang, Wu Wenqi, Zhou Han, Wu Yuanyuan, "Real-Time Lane-Vehicle Detection and Tracking System", IEEE,2016.

[21] Seongrae Kim, Junhee Lee, Youngmin Kim, "Speed-Adaptive Ratio- Based Lane Detection Algorithm for Self Driving Vehicles", IEEE,2016.

[22] Saina Ramyar, Abdollah Homaifar, Ali Karimoddini, Edward Tunstel, "Identification of Anomalies in Lane Change Behavior Using One-Class SVM", 2016 IEEE International Conference on Systems, Man, and Cybernetics SMC 2016 Budapest, Hungary,October 912,2016 .

[23] C. Zheng, C. Libo, Y. Linbo, Q. Qin, Z. Ruifeng, "Lane-Level Positioning System Based on RFID and Vision", 2016

[24] Mrinal Haloi, Dinesh Babu Jayagopi, "A Robust Lane Detection and Departure Warning System", 2015.

[25] Jung-Hwan Kim, Sun-Kyu Kim, Sang-Hyuk Lee, Tae-Min Lee, Joonhong Lim, "Lane Recognition Algorithm using Lane Shape and Color Features for Vehicle Black Box". 